\title{
SYRACUSE - A MULTI-JUNCTION CONCENTRATOR SYSTEM COMPUTER MODEL
}

\author{
N.J.Ekins-Daukes ${ }^{1}$, T.R.Betts ${ }^{2}$, Y.Kemmoku ${ }^{3}$, K.Araki ${ }^{4}$, H.S.Lee ${ }^{1}$, R.Gottschalg ${ }^{2}$, M.B.Boreland ${ }^{2}$, D.G.Infield ${ }^{2}$, \\ M.Yamaguchi ${ }^{1}$ \\ 1. Toyota Technological Institute, 2-12-1 Hisakata, Tempaku-ku, Nagoya, 468-8511, Japan \\ 2. University of Loughborough, U.K. \\ 3. Toyohashi Sozo College, Toyohashi, Japan \\ 4. Daido Steel Co. Ltd., Nagoya, Japan
}

\begin{abstract}
A multi-junction concentrator system computer model is presented, that aims to accurately model the power generated based on environmental data input, such as the irradiance, air temperature, humidity and pressure. The device model is discussed, illustrating the importance of statistical variation of module components when simulating a multi-cell module. This comprehensive concentrator computer model will be released for general use, aiming to become a useful resource for those designing and testing multi-junction based concentrator systems.
\end{abstract}

\section{Introduction}

Highly efficient III-V multi-junction solar cells have recently been incorporated into terrestrial solar concentration systems, promising high power density and potentially reducing the cost of photovoltaic power generation[1]. Field tests are currently underway on such concentrator modules at two locations in Japan, Inuyama and Toyohashi University of Technology [2]. Regular measurements of the system power output, IV profile and solar irradiation are made, together with meteorological measurements such as temperature, humidity and wind speed. Various module configurations are under test, but in this paper the discussion is restricted to a 550X module, composed of a Fresnel lens followed by a homogenizer to give a concentration ratio of 550 suns at the photovoltaic cell surface. The triple-junction InGaP/In ${ }_{0.01} \mathrm{GaAs} / \mathrm{Ge}$ solar cell attains an efficiency in excess of $38.9 \%$ for concentration ratios between 100 and 500 suns [1] and has been incorporated into a 20 cell module with a maximum module efficiency of $28.9 \%$ [3].

However the behaviour of these multi-junction modules in the field is sometimes complex and it can be difficult to extract detailed information regarding the performance of the concentrator system simply by inspecting the data. To obtain a greater understanding of the concentrator system in the outdoor environment, a computer model has been developed that simulates the concentrator system under realistic conditions. The computer model serves as a tool for evaluating particular concentrator system designs, identifying problems as they become apparent, as well as offering the possibility for calculating the annual power output under particular climatic conditions. In particular the spectral irradiance, PV cell temperature effects and statistical variation in the module components must all be accurately modelled if the module power output is to be correctly predicted. In this paper, the device and module modelling is described, extending the discussion presented at a previous conference[10].

\section{Description of the Syracuse computer model}

The computer model, called Syracuse, is broadly composed of four components and is illustrated in figure 1. The first is a spectral irradiance model that accounts for climatic variation in the sunlight, giving a realistic estimate of the spectral photon flux incident on the concentrator module. The second component is a photovoltaic device model that simulates the photogeneration and recombination in each of the component junctions in the multi-junction solar cell. The third component of the model assembles the photovoltaic device models into a circuit network representing the module and system configuration. Finally, the forth component solves the PV circuit network and the results are collected into a form in which they can be readily compared with the outdoors test data.

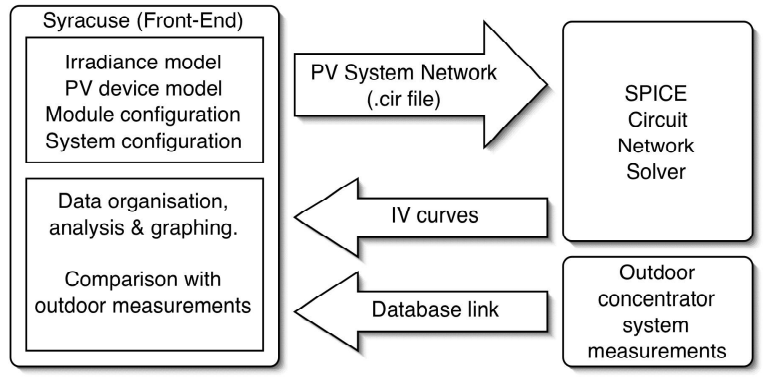

Fig.1. Schematic diagram showing how the Syracuse front end links with the SPICE circuit network solver and the outdoor measurement database.

The Syracuse model is written in the Java programming language and is designed to be easy to use and the user 
input takes place through a series of forms and windows, making it straightforward to inspect parameters and set up simulations. All the user inputs are passed as appropriate netlist files to the freely available SPICE circuit network solver [5]. The resulting IV curves are returned to the Syracuse program for further analysis, graphing and comparison with experimental data. The experimental data and outdoor test conditions are held in a MySQL database, accessible across the internet. The general process is shown in figure 1.

The database holds both instantaneous and averaged measurements, employing the following data filtering scheme. Measurements of direct normal irradiance (DNI), global irradiance $(\mathrm{Gl})$, module temperature, ambient temperature, humidity and a full IV curve are taken every $10 \mathrm{~s}$ at the test site. First a decision is made regarding the suitability of the data. If only the $\mathrm{GI}$ is greater than 10 W. $\mathrm{m}^{-2}$, the environmental data and $\mathrm{GI}$ is accepted but not the module IV data. Once the DNI is greater than $10 \mathrm{~W} . \mathrm{m}^{-}$ 2 the full IV data is accepted. Accepted data is then passed through a 3-point filter, where the median value of the three data points is selected as representative. The purpose of this filter is to eliminate spikes in the data that typically disrupt one measurement. The data that emerges from the 3-point filter is then averaged over a 5 minute interval. All parameters except humidity and IV curve data are averaged. A non-averaged measurement and associated IV curve is selected at the time closest to the mid-point of the 5 minute time interval. Both the averaged and representative non-averaged measurement are stored in the database. This scheme therefore offers the advantages of accuracy and data reduction afforded by averaging data, but preserves one set of instantaneous data during the 5 minute interval for a meaningful IV curve comparison. This scheme can also be used to detect the presence of transient phenomena, such as varying DNI or temperature by comparing the averaged values with that of the non-averaged 'representative' measurement.

A simple equivalent circuit for the multi-junction solar cell is used, each sub-cell composed of a current source, double diode recombination diodes corresponding to the radiative and non-radiative recombination and appropriate series and shunt resistances. In addition the cell is protected by a bypass diode.. The diode/resistance parameters were obtained from extensive testing of individual sub-cells[6] at $25^{\circ} \mathrm{C}$.

The photogeneration rates are calculated by integrating the convolved spectral irradiance and quantum efficiency and lens losses. In turn, the quantum efficiency can either be fixed using an experimental measurement or calculated dynamically from the absorption coefficient as a function of cell temperature, junction thickness, diffusion coefficient etc. according to the standard solution to the drift-diffusion equations in a p-n junction [7].
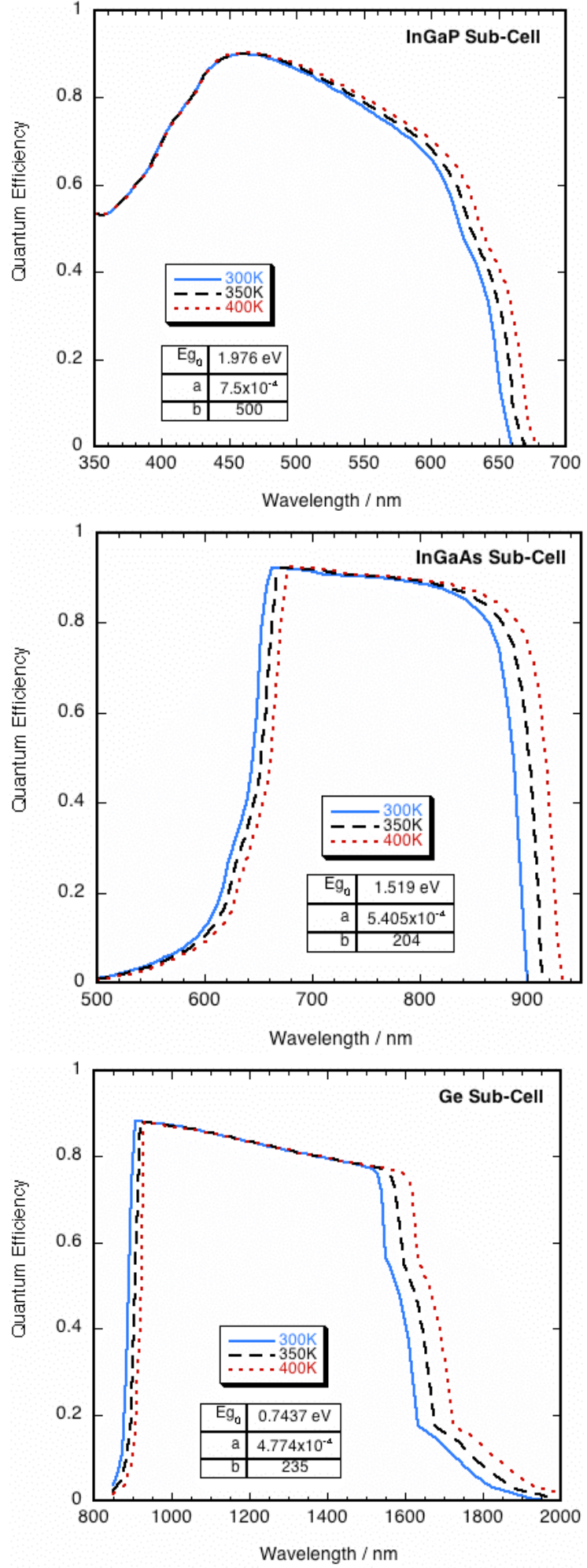

Fig. 2 QE profiles for the InGaP, InGaAs \& Ge sub-cells calculated as a function of temperature. 
Figure 2 shows the experimental and calculated quantum efficiency for all three sub-cells. In each case the calculation at $25^{\circ} \mathrm{C}$ fits the experimental data, while the broken line indicates the QE at the typical cell operating temperature of $70^{\circ} \mathrm{C}$. The absorption coefficient is assumed to shift in the same manner as the fundamental band-gap as described by equation 1 below:

$$
E g(T)=E g_{0}-\frac{a T^{2}}{b+T}
$$

where $\mathrm{Eg} 0$ is the band-gap at $\mathrm{OK}$ and $\mathrm{a}, \mathrm{b}$ are empirical coefficients. For simplicity, the mobility and surface recombination velocity were assumed to be independent of temperature.

The calculated QE broadly agrees with earlier experimental studies [8], giving confidence that the model gives a realistic estimate for the photogeneration in the multi-junction solar cell. The InGaP and InGaAs absorption coefficients were calculated from the model dielectric function (MDF) approach of Adachi [9]. The QE profiles shown in figure 2 are calculated assuming completely disordered InGaP, but in practice a small empirical adjustment to the absorption coefficient is required to account for compositional ordering that commonly occurs in the InGaP junction. The $\mathrm{Ge}$ absorption coefficient was taken from experimental data [10].

A typical concentrator module usually consists of many multi-junction receivers connected in series. While it is computationally advantageous to assume each cell in the module is similar, this leads to inaccurate results. Therefore all the cells in the module can be simulated with a Gaussian Isc distribution to account for cell and module manufacturing tolerances.

\section{Calculation With Experimentally Measured Spectral Irradiance.}

The spectral irradiance was measured on the $16^{\text {th }}$ September 2004 at $12: 16$ and is shown in figure 3 . The data was used to calculate the sub-cell photocurrents and generate the module netlist for a 550X concentrator module design consisting of $20 \mathrm{InGaP} / \mathrm{InGaAs} / \mathrm{Ge}$ multijunction cells connected in series, uniformly illuminated using dome shaped Fresnel lenses and a glass homogeniser [11].

The appropriate module temperature was extracted from the database and a $20^{\circ} \mathrm{C}$ offset added to obtain the cell temperature. The calculated IV curve was then compared with experimental IV test data taken almost simultaneously. The results of the calculation are shown if figure 4 and show a remarkably good fit to the experimental data. In particular, there is a gradual loss in current as the module bias is increased due to current mismatching in the module. If each series connected cell is assumed to be identical, the IV-curve is almost completely flat between 0 to $50 \mathrm{~V}$. However, introducing some random variation in the Isc leads to the observed slow degradation in current resulting in a lower fill factor. This behaviour is simply due to the bypass diodes which shunt the poorly performing cells at low module biases. At higher module biases the poorly performing cells become forward biased, so the bypass diodes no longer shunt the poorly performing cells, and the overall module current drops. Only a small distribution in short-circuit currents is required to give this effect, as noted in a prior study [4].

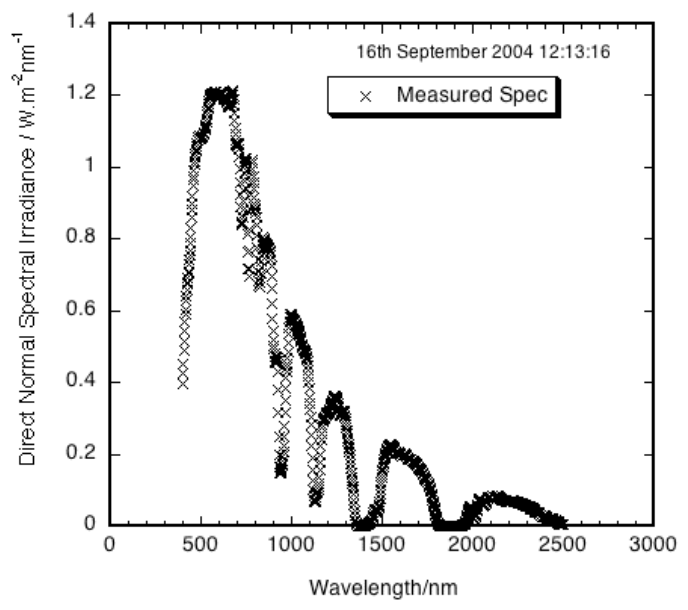

Fig. 3 Direct Normal Spectral Irradiance measured on the $16^{\text {th }}$ September 2004 at the Toyohashi test site.

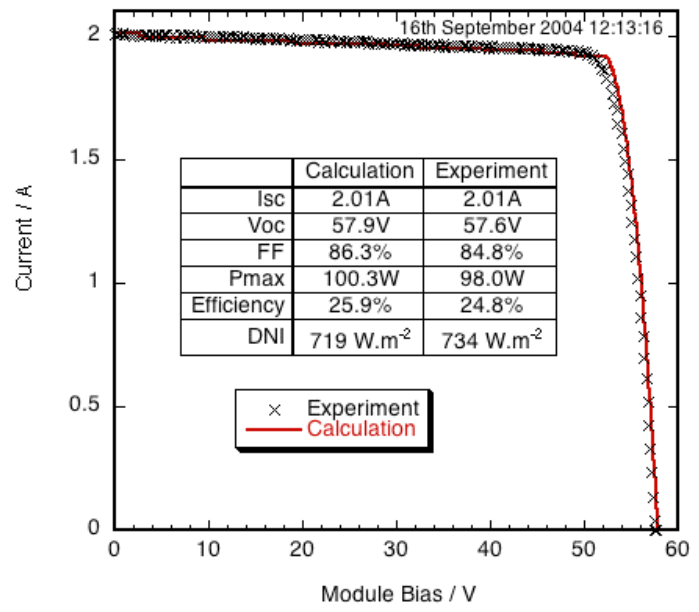

Fig.4 Comparison between the calculated IV curve derived from the spectral irradiance shown in figure 3 and a simultaneous experimental module IV measurement.

The excellent agreement between the model and experimental data demonstrates that if the spectral irradiance is known or can be calculated accurately, then a reliable calculation of the concentrator module power output can be made. The subject of accurately modelling the direct spectral irradiance is currently under study.

\section{Calculating the Module Temperature Coefficient}

The temperature coefficient of the concentrator module is a particularly difficult parameter to measure experimentally, primarily due to the fact that all testing 
must take place outdoors under largely uncontrolled conditions [12]. It was therefore considered desirable that the temperature coefficient be calculated theoretically as a reference for experimental attempts. As described earlier, the change in Jsc with temperature was accounted for by calculating the shift in band-gap energy with temperature and shifting the absorption coefficient accordingly, then deriving the quantum efficiency. The Voc dependence on temperature was accounted by considering the increase in the intrinsic carrier concentration $n_{i}$ with temperature [13]. This approach reproduces the temperature coefficient for all the photovoltaic parameters established in the previous study under 1-sun illumination [14]. The result is summarised in figure 5 below, showing the normalised efficiency temperature coefficient plotted against concentration. The relative temperature coefficient falls logarithmically with increasing concentration, yielding approximately half the 1 sun value at $550 \mathrm{X}$. This is a particularly important consideration when evaluating module performance, as the efficiency should be corrected to $25^{\circ} \mathrm{C}$. A comparison between this calculated trend and the measured experimental data can be found in another paper at this conference[3].

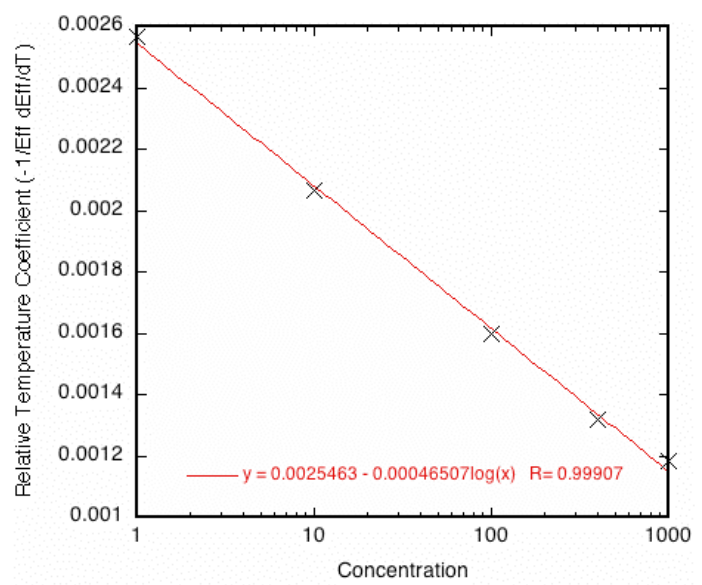

Fig.5 Relative temperature coefficient plotted as a function on concentration.

\section{CONCLUSION}

The Syracuse computer model allows users to analyse the performance of multi-junction concentrator systems and generic multi-junction devices. Recent validation tests have shown that accurate simulations can be performed and it is hoped that the model will become a valuable tool for designing multi-junction concentrator systems, investigating unusual concentrator system behaviour and for improving the design of multi-junction concentrator modules. The model will be offered to the photovoltaic community by mid-2005 and will be available from the web-site: www.syracuse-pv.webhop.org

\section{REFERENCES}

[1] M. Yamaguchi et al., "Japanese activities of R\&D on III-V concentrator solar cells and modules",Proc. 19th European Photovoltaic Solar Energy Conference, 2004, p. 2014-2017.

[2] Y. Kemmoku et al., "Field test of a concentrator photovoltaic system with flat Fresnel lens",Proc. 3rd World Conference and Exhibition on Photovoltaic Energy Conversion, 2003.

[3] K.Araki et al., "Comparison of efficiency measurements for a HCPV module with $3 \mathrm{~J}$ cells in 3 sites", Proc $31^{\text {st }}$ IEEE PVSC, 2005.

[4] N. J. Ekins-Daukes et al., "The design specification for Syracuse; a multi-junction concentrator system computer model",Proc. 19th European Photovoltaic Solar Energy Conference, 2004, p. 2466-2469.

[5]. SPICE Homepage http://bwrc.eecs.berkeley.edu/Classes/lcBook/SPICE/ [6] K. Nishioka et al., Jpn.J.Appl.Phys. 43, 882-889 (2004). [7] Jenny Nelson, The Physics of Solar Cells, (2003) Imperial College Press, London, U.K.

[8] D. Aiken et al., "Temperature dependent spectral response measurements for III-V multi-junction solar cells",Proc. 29th IEEE PV Specialists Conference, 2002 (IEEE), p. 828-831.

[9] S. Adachi, J.Appl.Phys. 66 6030-6040 (1989)

[10] E.D. Palik, "Handbook of optical Constants", (1998)

Academic Press, London, U.K.

[11] K.Araki et al., "Development Of A New 550x Concentrator Module With 3J Cells - Performance And Reliability" Proc $31^{\text {st }}$ IEEE PVSC (2005).

[12] Y. Kemmoku et al., "Modelling of module temperature of a concentrator PV system",Proc. 19th European

Photovoltaic Solar Energy Conference, 2004, p. 25682571.

[13] P. A. G. Massobrio, Semiconductor Device Modeling with SPICE, 2nd ed. (McGraw Hill, 1993)

[14] D. J. Friedman, "Modelling of tandem cell temperature coefficients",Proc. 25th IEEE PVSC, 1996, p. 89-92.

\section{Acknowlegements}

This work was supported by the Incorporated Administrative Agency New Energy and Industrial Technology Development Organization (NEDO) under Ministry of Economy, Trade and Industry (METI). and partially supported by the Ministry of Education, Culture, Sports, Science and Technology as a Private University Academic Frontier Research Center Program "Super High-Efficiency Photovoltaic Research Center". N.J.E-D gratefully acknowledges the support of a Japan Society for the Promotion of Science (JSPS) research fellowship. 